九州大学学術情報リポジトリ

Kyushu University Institutional Repository

\title{
ON THE OPTIMAL PARKING PROBLEM IN WHICH SPACES APPEAR RANDOMLY
}

Sakaguchi, Minoru

Department of Applied Mathematics, Faculty of Engineering Science, Osaka University

Tamaki, Mitsushi

Faculty of Economics, Otemon-gakuin University

https://doi.org/10.5109/13332

出版情報 : Bulletin of informatics and cybernetics. 20 (1/2), pp.1-10，1982-03. Research Association of Statistical Sciences

バージョン :

権利関係 : 


\title{
ON THE OPTIMAL PARKING PROBLEM IN WHICH SPACES APPEAR RANDOMLY
}

\author{
By
}

\section{Minoru SaKagUCHI* and Mitsushi TAMAKI**}

(Received October 13, 1981)

\begin{abstract}
A motorist drives his car along a one-way street toward his destination and looks for a parking place. If he finds an unoccupied parking place, he must decide either park there and walk the distance to his destination or continue driving expecting to find another opportunity of parking nearer to the destination. Unoccupied parking places are assumed to appear in accordance with a Poisson process along the street. Also the location of the destination is a random variable with a known probability distribution. The objective of the optimal parking problem is to find a parking policy which minimizes the expected time to reach the destination. We derive the optimal parking policy and the minimum expected cost is obtained. Moreover a two-person game version is treated in which the objective of each player is to maximize the probability of being the first to reach the destination common to both players. The equilibrium strategy is derived explicitly.
\end{abstract}

\section{Introduction}

The parking problem was first presented by DeGroot [4; page 384]. A continuoustime version of the problem which we shall analyse in this paper can be described as follows: A motorist is driving his car along a one-way street toward his destination and is looking for a parking place. If he finds an unoccupied parking place, he must decide either park there and walk the distance to his destination or continue driving expecting to find another opportunity of parking nearer to the destination. Unoccupied parking places are assumed to appear in accordance with a Poisson process with arrival rate $\lambda$ along the street. Also the location of the destination is a random variable with a known cdf $F(x), x \geqq 0$.

Let $r \in[0,1]$ represent the inverse of the relative speed of driving a car (compared with one on foot). Then the time duration spent to reach the destination is measured by

$$
r \cdot(\text { Distance by driving })+(\text { Distance on foot }) \text {. }
$$

The objective of the decision problem is to find a parking policy which minimizes the

* Department of Applied Mathematics, Faculty of Engineering Science, Osaka University, Toyonaka, Osaka.

** Faculty of Economics, Otemon-Gakuin University, Ibaraki, Osaka. 
expected time to reach the destination.

In Section 2 we formulate the problem as an optimal stopping problem without recall. Since the underlying Markovian decision process satisfies the condition of the so-called "monotone case" (Chow, Robbins and Siegmund [2]) we shall derive an optimal stopping policy as an OLA stopping principle. The expected time to reach the destination under the optimal parking policy is also obtained.

In Section 3 we consider a two-person game version of the parking problem. The objective of each player in this game is to maximize the probability of being the first to reach the destination common to both players. The equilibrium strategy is derived explicitly.

\section{Optimal Parking Problem and Its Solution}

Our problem is an optimal stopping problem without recall. Define the state $x$ to be the situation, in which the driver has driven $x$ units of distance without passing through the destination, and an unoccupied parking place is just arrived at. If he has passed by the destination without having parked his car before this instant, his best decision hereafter is evidently to park at the first opportunity arrived since then and walk backwards to the destination. Hence we have only to find how to decide in state $x$ defined above. Denote by $V(x)$ the expected value of (1.1) obtainable by following an optimal policy starting from the state $x$.

Since we are assuming perfect detection of the destination, the posterior distribution of its location has pdf:

$$
f(t) / \bar{F}(x) \text {, for } t>x \text {; and } 0 \text {, for } t<x .
$$

where $F^{\prime}(x)=f(x)$ and $\bar{F}(x)=1-F(x)$. Hence the "stop cost" at state $x$ is

$$
g(x) \equiv \int_{x}^{\infty}(t-x) d F(t) / \bar{F}(x)=T_{F}(x) / \bar{F}(x),
$$

where $T_{F}(x) \equiv \int_{x}^{\infty}(t-x) d F(t)$ is the well-known mean-shortage function (cf. DeGroot [4; Section 13.4]). The "continuation outcome" at state $x$ is derived by considering which of the two possible events, the next opportunity (abbr. by $O$ ) and the detection of the destination (abbr. by $D$ ) comes earlier, i.e.,

$$
\begin{aligned}
P V(x)= & \int_{0}^{\infty}(r s+V(x+s)) \bar{F}(x+s) \lambda e^{-\lambda s} d s / \bar{F}(x) \\
& +\int_{x}^{\infty}\left\{r(t-x)+(r+1) \lambda^{-1}\right\} f(t) e^{-\lambda(t-x)} d t / \bar{F}(x) \\
= & \int_{0}^{\infty} V(x+s) \bar{F}(x+s) \lambda e^{-\lambda s} d s / \bar{F}(x)+k(x), \quad \text { say. }
\end{aligned}
$$

A bit of computation involving integration by parts gives

$$
k(x) \equiv \int_{0}^{\infty}\left[r s \bar{F}(x+s)+\left\{\lambda^{-1} r s+\lambda^{-2}(r+1)\right\} f(x+s)\right] \lambda e^{-\lambda s} d s / \bar{F}(x)
$$




$$
=\lambda^{-1} r+\int_{x}^{\infty} e^{-i\langle s-x\rangle} f(s) d s /(\lambda \bar{F}(x)),
$$

and we thus arrived at the optimality equation

$$
V(x)=\min \{g(x), P V(x)\} .
$$

The optimal stopping problem we are treating is a Markov version of the monotone case of Chow-Robbins-Siegmund [2; Section 3.5] and it can be solved by an application of the one-stage look-ahead (abbr. by OLA) policy, provided some regularity conditions are satisfied by the decision process. Let

$$
B \equiv\{x \geqq 0 \mid g(x) \leqq P g(x)\},
$$

i.e., the set of states at which stopping immediately is at least as good as continuing for exactly one more transition and then stopping. It is known that if $B$ is "realizable", i.e., Pr. \{state eventually enters $B\}=1$, and "closed", i.e., Pr. \{leave $B$ at some finite time $\mid$ currently in $x \in B\}=0$, then the first hitting time of $B$ is the optimal stopping time. For rigorous proofs, see for instance Bojdecki [1] and Cowan and Zabczyk [3].

We shall prove the following results.

LEMMA 2.1 The OLA stopping region $B$ is given by

$$
B=\left\{x \geqq 0: \phi(x) \equiv \int_{x}^{\infty} e^{-\lambda(t-x)} d F(t) / \bar{F}(x) \geqq(1-r) / 2\right\} .
$$

Proof. From (2.2) and (2.3)

$$
g(x) \leqq P g(x) \Longleftrightarrow T_{F}(x) \leqq \int_{0}^{\infty} T_{F}(x+s) \lambda e^{-\lambda s} d s+\bar{F}(x) k(x) .
$$

Furthermore, straightforward calculation gives

$$
\int_{0}^{\infty} T_{F}(x+s) \lambda e^{-\lambda s} d s=T_{F}(x)-\lambda^{-1} \int_{x}^{\infty}\left(1-e^{-\lambda(t-x)}\right) d F(t) .
$$

(For a proof use $T_{G}(z)=\lambda^{-1} e^{-i z}(z>0) ;=\lambda^{-1}-z(z<0)$, if $d G(x) / d x=\lambda e^{-i x}$ ). Substituting these facts into (2.6) we obtain (2.7).

Note that $\phi(x)$ is the conditional probability that $D$ comes earlier than $O$, given that the process is currently in state $x$. We thus have

Proposition 2.2. If the region $B$ given by (2.7) is a non-empty interval $\left[x^{*}, A\right.$ ), where $A$ may be infinity, then it is an optimal stopping region.

Hence if the above condition is satisfied it is optimal for the driver to park at the first opportunity arrived after he drives to the state $x^{*}$ or after detection of the destination, whichever comes earlier, where $x^{*}$ is a unique root of the equation

$$
\dot{\phi}(x) \equiv \int_{x}^{\infty} e^{-\lambda(t-x)} d F(t) / \tilde{F}(x)=(1-r) / 2,
$$

or zero (in case of non-existence of the root).

Table 1 below presents the corresponding $\phi(x)$ and the critical level $x^{*}$ for the various location distribution $F(x)$ for which the set $B$ becomes a non-empty interval $\left[x^{*}, A\right)$.

Proposition 2.3. Let $B$ given by (2.7) be an optimal stopping region. Then 
Table 1

Distribution of Destination

(a) Deterministic :

$$
F(x)=1(x \geqq 1) ;=0(0 \leqq x<1) . \quad \phi(x)=e^{-\dot{x}(1-x)}
$$

(b) Uniform:

$$
F(x)=x,(0 \leqq x \leqq 1) . \quad \phi(x)=\left[1-e^{-\lambda(1-x)}\right] / \lambda(1-x)
$$

(c) Triangular:

$$
F(x)=x^{2},(0 \leqq x \leqq 1) . \quad \quad \phi(x)=2 \lambda^{-1}\left[\lambda^{-1}+x-\left(1+\dot{\lambda}^{-1}\right) e^{-\dot{\lambda}(1-x)}\right] /\left(1-x^{2}\right)
$$

(d) Gamma :

$$
F(x)=1-(1+x) e^{-x}, \quad(x \geqq 0), \quad \phi(x)=(1+\lambda)^{-1}\left\{(1+\lambda)^{-1}+x\right\} /(1+x)
$$

with the condition $(1-r) / 2<(1+\lambda)^{-1}$.

$$
V(x)=\left\{\begin{array}{cc}
g\left(x^{*}\right) \bar{F}\left(x^{*}\right) / \bar{F}(x)+\lambda^{-1}(1+r)\left(1-\bar{F}\left(x^{*}\right) / \bar{F}(x)\right) & \\
+r\left(T_{F}(x)-T_{F}\left(x^{*}\right)\right) / \bar{F}(x), & x<x^{*} \\
g\left(x^{*}\right), & x>x^{*} .
\end{array}\right.
$$

Proof. From Proposition 2.2 and (2.5) we have

$$
V(x)=\left\{\begin{array}{rr}
P V(x), & x<x^{*} \\
g(x), & x \geqq x^{*} .
\end{array}\right.
$$

For $x<x^{*}$ we have from (2.3) and (2.4).

$$
\left.V(x)=P V(x)=\int_{x}^{\infty} V(s) \bar{F}(s) \lambda e^{-\lambda(s-x}\right) d s / \bar{F}(x)+\lambda^{-1}(r+\phi(x)) .
$$

Multiplying both sides by $\bar{F}(x)$, and differentiating in $x$, we obtain the differential equation

$$
V^{\prime}(x)=\left(V(x)-\lambda^{-1}(1+r)\right) f(x) / \bar{F}(x)-r .
$$

Integration gives

$$
\left(V(x)-\lambda^{-1}(1+r)\right) \bar{F}(x)=r T_{F}(x)+c,
$$

where the integration constant $c$ is explicitly determined by the relation $V\left(x^{*}\right)=g\left(x^{*}\right)$. This gives the desired result.

Let us check the validity of the equation (2.9) as follows. Define the z-level strategy for the driver as the one by which he parks his car at the first opportunity arrived after he drives to a fixed location $z \in[0, \infty)$, or after detection of the destination, whichever comes earlier. The expected cost obtainable by following the $z$-level strategy, is defined to be the expected time until he reaches to the destination by following the strategy, and will be denoted by $v(z)$.

Proposition 2.4. Suppose that the pdf satisfies

$$
\phi(z) \geqq\left(\frac{1+r}{2 \lambda}\right) f(z) / \bar{F}(z)
$$


for all $z \geqq 0$ with $\bar{F}(z)>0$. Then the expected cost $v(z)$ is minimized at $z=x^{*}$, where $x^{*}$ is a unique root of the equation (2.8). Moreover we have

$$
\min _{z \geq 0} v(z)=(1-r) \vec{F}\left(x^{*}\right) g\left(x^{*}\right)+\lambda^{-1}(1+r) F\left(x^{*}\right)+r \mu .
$$

where $\mu \equiv \int_{0}^{\infty} t d F(t)$.

Proof. By the definition of the $z$-level strategy we have

$$
\begin{aligned}
v(z)= & \int_{0}^{z}\left\{r\left(t+\lambda^{-1}\right)+\lambda^{-1}\right\} f(t) d t \\
& +\int_{z}^{\infty} f(t) d t\left[\left\{\int_{z}^{t}(r s+t-s)+\int_{t}^{\infty}(r s+s-t)\right\} \lambda e^{-\lambda(s-z)} d s\right] .
\end{aligned}
$$

In the r.h.s. of the expression, the first (second) term represents the expected cost when $D$ comes earlier (later) than driving to the location $z$. The whole expression of $v(z)$ is, after a bit of calculation involving integration by parts, reduced to

$$
v(z)=\lambda^{-1}(1+r)+r \mu+(1-r) T_{F}(z)-2 \int_{z}^{\infty} e^{-\lambda(t-z)} \bar{F}(t) d t .
$$

We thus have

$$
v^{\prime}(z)=(1+r) \bar{F}(z)-2 \int_{z}^{\infty} e^{-\lambda(t-z)} \bar{F}(t) d t
$$

and

$$
v^{\prime \prime}(z)=-(1+r) f(z)+2 \lambda \int_{z}^{\infty} e^{-\lambda(t-z)} f(t) d t .
$$

The condition $v^{\prime}(z)=0$ gives

$$
\begin{aligned}
(1+r) \bar{F}(z) & =2 \int_{z}^{\infty} \lambda e^{-\lambda(t-z)} \bar{F}(t) d t \\
& =2\left[\bar{F}(z)-\int_{z}^{\infty} e^{-\lambda(t-z)} d F(t)\right],
\end{aligned}
$$

which reduces to

$$
\phi(z) \equiv \int_{z}^{\infty} e^{-\lambda(t-z)} d F(t) / \bar{F}(z)=(1-r) / 2
$$

The condition stated in the proposition guarantees $v^{\prime \prime}(z) \geqq 0$. If we substitute (2.13) into (2.12) then we obtain (2.11). This completes the proof.

From (2.9) we have

$$
\begin{aligned}
V(0+) & =g\left(x^{*}\right) \vec{F}\left(x^{*}\right)+\lambda^{-1}(1+r) F\left(x^{*}\right)+r\left(\mu-T_{F}\left(x^{*}\right)\right) \\
& =(1-r) g\left(x^{*}\right) \vec{F}\left(x^{*}\right)+\lambda^{-1}(1+r) F\left(x^{*}\right)+r \mu
\end{aligned}
$$

since $T_{F}\left(x^{*}\right)=g\left(x^{*}\right) \bar{F}\left(x^{*}\right)$. Hence Proposition 2.4 gives $V(0+)=\min _{z \geqq 0} v(z)$, if the stated condition is satisfied by the pdf.

REMARK 1. An important case in which the condition (2.10) is satisfied is Example (a) in Table 1. In this case we have from (2.11), 


$$
V(0+)=\min _{z \geq 0} v(z)=(1-r)\left(1-x^{*}\right)+r=r x^{*}+1-x^{*}
$$

and $x^{*}$ is given by

$$
x^{*}=\left[1-\lambda^{-1} \log (2 /(1-r))\right]^{+} .
$$

We can interpret this result as: The critical level of the optimal parking policy is determined such that it makes the expected time spent by following the optimal policy identical to $r^{*} x+1-x^{*}$, i.e., the time spent if we drive to $x^{*}$ and park there as if empty parking places were accessible everywhere along the street.

\section{A Two-Person Game Related to the Parking Problem}

In this section we consider a two-person game version of the parking problem in which the situation can be described as follows: There are two motorists each of whom is driving along a disparate one-way street toward a common destination and is looking for a parking place. Each motorist considers his decision problem in the conditions of the parking problem analysed in Section 1 in the case of degenerate location distribution of the destination, i.e., $F(x)=1,(x \geqq 1) ;=0,(0 \leqq x<1)$. We assume that the Poisson processes for the players proceed independently with the identical arrival rate $\lambda$. We call a "win" for each player the event in which he reaches the destination earlier than his opponent. If the two players reach the destination simultaneously, both of them are the winners. The goal of each player in the game is to maximize the probability that he becomes a single winner.

A strategy which prescribes parking at the first opportunity arrived after driving $x$ units of distance will be called $x$-level strategy and will be denoted by $\sigma_{x}$. The importance of the $x$-level strategies for a broad class of the optimal stopping problems is widely known (see, e.g. DeGroot [4; Chapter 13]).

Suppose that players I and II employ the strategy $\sigma_{x}$ and $\sigma_{y}$ with $0 \leqq x \leqq y \leqq 1$, respectively. We don't need consider level strategies with the level greater than 1 since they are uniformly dominated by the 1-level strategy. Denote by $M_{i}\left(\sigma_{x}, \sigma_{y}\right)$ player $i$ 's expected payoff, $i$.e. probability of win under the strategy pair $\sigma_{x}-\sigma_{y}$. Then, for $0 \leqq x \leqq y \leqq 1$, we have

$$
M_{1}\left(\sigma_{x}, \sigma_{y}\right)=\int_{x}^{y} \lambda e^{-\lambda(t-x)} \cdot e^{-\lambda\left(s_{+}(t)-y\right)} d t+\frac{1}{2} e^{-\lambda(y-x)} .
$$

In the r.h.s. of this equation, the first (second) term is the probability of I's win conditioned that the first opportunity for I after he drives $x$ units of distance arrives before (after) driving to the location $y$. In the first term I's conditional win occurs only when II drives at least a distance of

$$
s_{+}(t) \equiv \frac{2-(1-r) t}{1+r} \quad(\geqq 1 \text {, for all } r \in[0,1) \text { and } t \in[0,1])
$$

and then walking back to the destination, if I's parking location is at $t \in(x, y)$ This is readily obtained by solving the equation

$$
1-t=r(s-t)+s-1
$$


In the second term in (3.1) we used the fact that if I's opportunity for parking has not arrived until he drives to $y$, the game reduces to the initial situation, with unit distance replaced by $1-y$ units of distance, but with structural symmetry in the game unchanged.

The payoff to I for $0 \leqq y \leqq x \leqq 1$ can be found at once from the symmetry and constant-sum property (sum is unity) of the game. That is,

$$
M_{1}\left(\sigma_{x}, \sigma_{y}\right)=M_{2}\left(\sigma_{y}, \sigma_{x}\right)=1-M_{1}\left(\sigma_{y}, \sigma_{x}\right),
$$

where the last expression reduces to (3.1).

The unique equilibrium values are $1 / 2,1 / 2$ for the players. We shall prove the following

Proposition 3.1. The common equilibrium strategy for each player is given by the $x^{0}$-level strategy, where

$$
x^{0}=[1-(1+r) \log 2 /(2 \lambda)]^{+} .
$$

Proof. To prove the proposition it is sufficient to show that

$$
\frac{\partial M_{1}\left(\sigma_{x}, \sigma_{x 0}\right)}{\partial x} \geqq 0, \quad \text { for } 0 \leqq x \leqq x^{0},
$$

and

$$
\frac{\partial M_{1}\left(\sigma_{x 0}, \sigma_{y}\right)}{\partial y} \geqq 0, \quad \text { for } x^{0} \leqq y \leqq 1 .
$$

Substituting (3.2) into (3.1) we obtain after some calculation

$$
M_{1}\left(\sigma_{x}, \sigma_{y}\right)=(2 c)^{-1} e^{-2 \lambda(1-c)}\left\{e^{\lambda((1-2 c) x+y)}-e^{\lambda(x+(1-2 c) y)}\right\}+(1 / 2) e^{-\lambda(y-x)},
$$

for $0 \leqq x \leqq y \leqq 1$, where $c \equiv r /(1+r)$.

Then noting that

$$
e^{\lambda x^{0}}=2^{-(1+r) / 2} e^{\lambda}, \quad \text { and } \quad e^{\lambda(1-2 c) x^{0}}=2^{-(1-r) / 2} e^{\lambda(1-2 c)}
$$

we can find after some simplification that

$$
\frac{\partial M_{1}\left(\sigma_{x}, \sigma_{x 0}\right)}{\partial x}=\left(r^{-1}-1\right) e^{-\lambda(1-x)}\left[2^{-(1+r) / 2} e^{2 \lambda c(1-x)}-2^{-(1-r) / 2}\right]
$$

for $0 \leqq x \leqq x^{0}$. The factor inside the bold brackets is non-negative if and only if $0 \leqq x \leqq x^{0}$ and equals zero at $x$ satisfying $e^{2 \lambda(1-x)}=2^{r}$, or

$$
x=1-r \log 2 /(2 \lambda c)=1-(1+r) \log 2 /(2 \lambda) .
$$

This shows the validity of (3.5).

Next we have to prove (3.6). We can easily find that $\partial M_{1}\left(\sigma_{x^{0}}, \sigma_{y}\right) / \partial y$, for $x^{0} \leqq y \leqq 1$, is identical to minus of r.h.s. of (3.8) with $x$ replaced by $y$. Hence the validity of (3.6) is evident. This completes the proof of the proposition.

In the rest of this section we shall treat a generalization to the non-symmetric case, where the opportunities for the players arrive with different rates $\lambda_{1}$ for I, and $\lambda_{2}$ for II. Consider the level-strategy-pair $\sigma_{x}-\sigma_{y}$, with $0 \leqq x \leqq y \leqq 1$. Let $O_{1}$ denote the first opportunity of parking for I after he drives his level distance, and $\mathrm{O}_{2}$ similarly for II. 
Then I can win in the following three cases: Case $1^{\circ}$. $O_{1}$ comes at $t \in(x, y)$, and $O_{2}$ comes in the interval $\left(s_{+}(t), \infty\right)$, where $s_{+}(t)$ is defined by (3.2). Case $2^{\circ}$. $O_{1}$ comes at $t \in(y, 1)$, and $O_{2}$ comes in $(y, t) \cup\left(s_{+}(t), \infty\right)$, and Case $3^{\circ} . O_{1}$ comes at $t \in(1, \infty)$, and $O_{2}$ comes in $\left(y, y \vee s_{-}(t)\right) \cup(t, \infty)$, where $s_{-}(t)$ is defined by

$$
s_{-}(t) \equiv \begin{gathered}
2-(1+r) t \\
1-r
\end{gathered} \quad(\leqq 1 \text {, for all } r \in[0,1) \text { and } t \in[1, \infty)) .
$$

Therefore the probability of I's win under the strategy-pair $\sigma_{x}-\sigma_{y}$ with $0 \leqq x \leqq y \leqq 1$ is

$$
\begin{aligned}
M_{1}\left(\sigma_{x}, \sigma_{y}\right) & =\int_{x}^{y} \lambda_{1} e^{-\lambda_{1}(t-x)} \cdot e^{\lambda_{2}\left(s_{+}(t)-y\right)} d t \\
& +\int_{y}^{1} \lambda_{1} e^{-\lambda_{1}(t-x)} d t\left[1-\int_{t}^{s_{+}(t)} \lambda_{2} e^{-\lambda_{2}(s-y)} d s\right] \\
& +\int_{1}^{s_{+}(y)} \lambda_{1} e^{-\lambda_{1}(t-x)} d t\left[1-\int_{s_{-}(t)}^{t} \lambda_{2} e^{-\lambda_{2}(s-y)} d s\right] \\
& +\int_{s_{+}(y)}^{\infty} \lambda_{1} e^{-\lambda_{1}(t-x)} \cdot e^{-\lambda_{2}(t-y)} d t .
\end{aligned}
$$

In the r.h.s. of this equation, the first (second) term corresponds to Case $1^{\circ}\left(2^{\circ}\right)$, and the remaining two terms correspond to Case $3^{\circ}$.

The payoff to I for $0 \leqq y \leqq x \leqq 1$ can be found from the constant-sum property of the game. That is, $M_{1}\left(\sigma_{x}, \sigma_{y}\right)$, for $0 \leqq y \leqq x$ is unity minus the r.h.s. of (3.10), with the interchanges $\lambda_{1} \leftrightarrow \lambda_{2}$ and $x \leftrightarrow y$.

PROPOSITION 3.2. Suppose that $\lambda_{1}<\lambda_{2}$ without loss of generality. Then if the simultaneous equations (3.18)-(3.19) mentioned later in the proof have a unique root $(\bar{u}, \bar{v})$, with $1 \leqq \bar{u} \leqq \bar{v} \leqq e$, then the unique equilibrium strategy-pair is given by $\sigma_{\bar{x}}-\sigma_{\bar{y}}$, where

$$
\bar{x}=1-\log \bar{u} \text { and } \bar{y}=1-\log \bar{v} .
$$

Moreover we have $M_{1}\left(\sigma_{\bar{x}}, \sigma_{\bar{y}}\right)=\left(\bar{u}^{\gamma} \bar{v}\right)^{-\lambda_{2}}$.

Proof. Substituting (3.2) and (3.9) into (3.10), and performing integrations and rearrangements, we finally obtain, for $0 \leqq x \leqq y \leqq 1$,

$$
\begin{aligned}
& M_{1}\left(\sigma_{x}, \sigma_{y}\right)=\frac{\lambda_{1} \lambda_{2}\left(\lambda_{2}-\lambda_{1}\right)(1+\gamma)^{2}}{\left(\lambda_{1}+\lambda_{2}\right)\left(\lambda_{1}-\gamma \lambda_{2}\right)\left(\gamma \lambda_{1}-\lambda_{2}\right)} e^{-\left\{\lambda_{1}(1-x)+\lambda_{2}(1-y)\right\}} \\
& +\frac{\lambda_{1}}{\lambda_{1}-\gamma \lambda_{2}} e^{\left.-\lambda_{2} \mid \gamma(1-x)+1-y\right)}+\frac{\lambda_{2}}{\gamma \lambda_{1}-\lambda_{2}} e^{-\lambda_{1}(1-x+\gamma(1-y)\}} \\
& +\frac{\lambda_{2}}{\lambda_{1}+\lambda_{2}} e^{-\lambda_{1}(y-x)}
\end{aligned}
$$

where $r \equiv(1-r) /(1+r)$. [If $\lambda_{1}=\lambda_{2}=\lambda$, (3.11) reduces to (3.7) since $\left.r=1-2 c\right]$. Hence

$$
\begin{aligned}
& \frac{\partial M_{1}\left(\sigma_{x}, \sigma_{y}\right)}{\partial x}=\frac{\lambda_{1} \lambda_{2} e^{-\left\{\lambda_{1}(1-x)+\lambda_{2}(1-y)\right\}}}{\left(\lambda_{1}+\lambda_{2}\right)\left(\lambda_{1}-\gamma \lambda_{2}\right)\left(\gamma \lambda_{1}-\lambda_{2}\right)}\left[\lambda_{1}\left(\lambda_{2}-\lambda_{1}\right)(1+\gamma)^{2}\right. \\
& +\gamma\left(\lambda_{1}+\lambda_{2}\right)\left(\gamma \lambda_{1}-\lambda_{2}\right) e^{\left(\lambda_{1}-\gamma \lambda_{2}\right)(1-x)}+\left(\lambda_{1}+\lambda_{2}\right)\left(\lambda_{1}-\gamma \lambda_{2}\right) e^{\left(\lambda_{2}-\gamma \lambda_{1}\right)(1-y)} \\
& \left.+\left(\lambda_{1}-\gamma \lambda_{2}\right)\left(\gamma \lambda_{1}-\lambda_{2}\right) e^{\left(\lambda_{1}+\lambda_{2}\right)(1-y)}\right] \text {. }
\end{aligned}
$$


Letting

$$
\left\{\begin{array}{l}
a=\lambda_{1}-\gamma \lambda_{2} \\
b=\lambda_{2}-\gamma \lambda_{1}
\end{array}, \quad\left\{\begin{array}{l}
u=e^{1-x} \\
v=e^{1-y}
\end{array}\right.\right.
$$

we can rewrite (3.12) as

$$
\begin{gathered}
\frac{\partial M_{1}\left(\sigma_{x}, \sigma_{y}\right)}{\partial x}=\frac{\lambda_{1} \lambda_{2} u^{-\lambda_{1}} v^{-\lambda_{2}}}{a b(a+b)} \\
{\left[(a-b)(a+\gamma b)+\gamma b(a+b) u^{a}-a(a+b) v^{b}\right.} \\
\left.+(1-\gamma) a b v^{(a+b) /(1-\gamma)}\right] .
\end{gathered}
$$

Similary we can obtain

$$
\begin{aligned}
\frac{\partial M_{1}\left(\sigma_{x}, \sigma_{u}\right)}{\partial y}=\frac{\lambda_{1} \lambda_{2} u^{-\lambda_{1}} v^{-\lambda_{2}}}{a b(a+b)} & {\left[(a-b)(b+\gamma a)+b(a+b) u^{a}\right.} \\
& \left.-\gamma a(a+b) v^{b}-(1-\gamma) a b v^{(a+b) /(1-\gamma)}\right] .
\end{aligned}
$$

Hence the first order conditions $\partial M_{1}\left(\sigma_{x}, \sigma_{y}\right) / \partial x=0$ and $\partial M_{1}\left(\sigma_{x}, \sigma_{y}\right) / \partial y=0$ are equivalent to

$$
(a-b)(a+\gamma b)+\gamma b(a+b) u^{a}-a(a+b) v^{b}+(1-\gamma) a b v^{(a+b) /(1-\gamma)}=0
$$

and

$$
(a-b)(\gamma a+b)+b(a+b) u^{a}-\gamma a(a+b) v^{b}-(1-\gamma) a b v^{(a+b) /(1-\gamma)}=0,
$$

respectively. Adding both equations side by side we get the equation

$$
a-b+b u^{a}-a v^{b}=0,
$$

which, if substituted into (3.16), gives

$$
a-b-(a+b) v^{b}+b v^{(a+b) /(1-r)}=0 .
$$

[If $\lambda_{1}=\lambda_{2}=\lambda$, then (3.18) and (3.19) reduce to a single equation $2 u^{(1-\gamma) \lambda}=u^{2 \lambda}$, which gives $x=1-\log u=1-(1+r) \log 2 /(2 \lambda)$, coinciding with (3.4)]. The rest of the proof can be done by the usual routine and will be omitted.

Some values of the levels in the equilibrium strategy-pairs are shown in Table 2.

Table 2

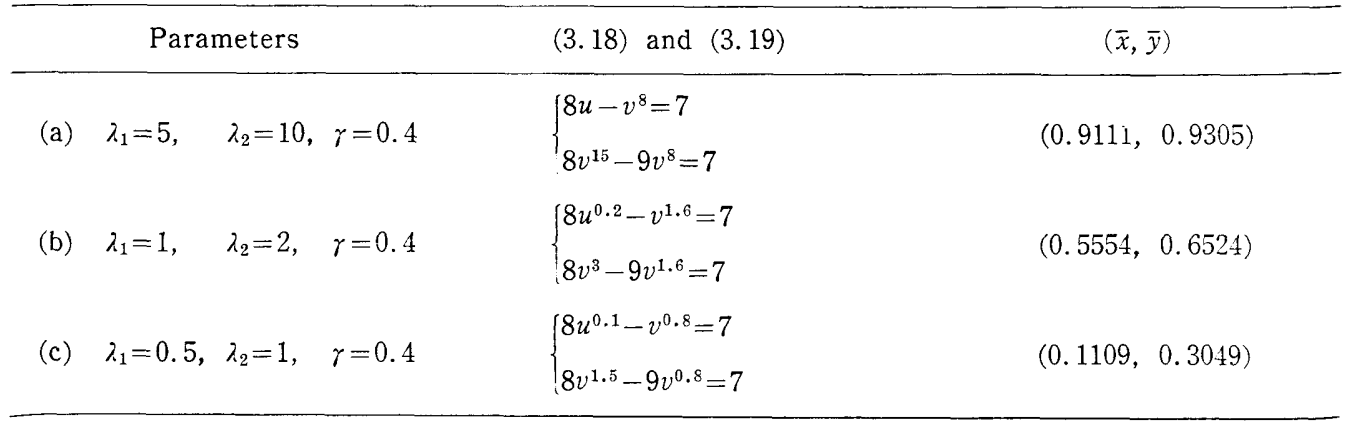

REMARK 1. The level $x^{0}$ of the equilibrium strategy given by (3.4) in the symmetric case does not coincide with the critical number $x^{*}$ of the corresponding parking problem 
(one-person game version) analysed in Section 2. By the result (a) in Table 1 we get

$$
x^{*}=\left[1-\lambda^{-1} \log (2 /(1-r))\right]^{+} .
$$

We easily see that $0<x^{*}<x^{0}<1$, if both are positive. This means that in the competitive situation each player becomes unprudent, that is, he does not park until he drives closer to the destination.

REMARK 2. The similar game situation as in ours associated with the optimal stopping are discussed by Domanskiy [5], Sakaguchi [6] and Styszynski [7]. It seems to the authors that the game model in which the common destination is randomly located as in Section 2 has some difficulty to set up its formulation.

\section{Acknowledgement}

The authors are grateful to Minoru Yoshida for his help to prepare Table 2.

\section{References}

[1] T. BOJDECKI: On optimal stopping of a sequence of independent randcm variables-Probability maximizing approach. Stoch. Process and Their Appl. 6 (1978), 153-163.

[2] Y. Chow, H. Robbins and D. Siegmexd: Great Expectations: The Theory of Optimal Stopping. Houghton Mifflin Company, Boston, 1971.

[3] R. CowAX and J. ZABCZYK: An optimal selection problem associated with the Poisson process. Theo. Prob. Appl. 23 (1978), 584-592.

[4] M.H. DeGroot: Optimal Statistical Decisions. McGraw-Hill Book Company, New York 1970.

[5] V.K. Domaxskiy: On certain games connected with a sequence of Bernoulli trials. Eng. Cybernetics, 12 (1974), 25-29.

[6] M. SAKAgLCHI: Non-zero-sum games related to the secretary problem. J. Oper. Res. Soc. Japan, 23 (1980), 287-293.

[7] A. Strszysisi: A silent-silent duel with bullets accessible at random moments of time. Working paper in Tech. Univ. Wroctow, 1979.

Communicated by T. Kitagawa 